\title{
High Frequency Spinal Cord Stimulation in a Patient with Bilateral COCHLEAR Implants
}

\author{
Ashwin Vaidyanathan, MD'1, Adam Romman, $\mathrm{MD}^{2}$, and Murali Patri, MBBS
}

Background: High-frequency spinal cord stimulation (HF-SCS) has become very popular in the management of chronic pain worldwide. As it relies on generating high-frequency electrical impulses, there is a risk of interference with other devices such as cochlear implants that utilize similar principles. A literature search did not reveal any case reports of HF-SCS implantation in a patient with cochlear implants.

Case Report: A 75-year-old White woman with a history of bilateral cochlear implants (Cochlear Americas Nucleus $®$ with cp910 processor) for severe sensorineural hearing loss presented to our chronic pain clinic with lumbosacral radiculopathy. The patient underwent a HF-SCS trial with entry point at the L1-L2 space and the leads positioned at the top and bottom of T8. The patient did not experience any auditory interference with her Cochlear implant at triple the average SCS stimulation strength. During the follow-up visit the next week, the patient reported nearly $80 \%$ symptomatic pain relief and significant functional improvement. There was no change in her hearing and no evidence of interference. The patient ultimately underwent percutaneous SCS paddle electrode placement and at 3 months, continues to have excellent pain relief without any auditory interactions.

Conclusion: We successfully implanted a HF-SCS at the thoracic level in a patient with bilateral cochlear implants without any auditory interference.

Key words: Cochlear implant, lumbar radiculopathy, spinal cord stimulation

\section{BACKGROUND}

Physicians around the world have been using spinal cord stimulation (SCS) for various chronic pain conditions since it was developed almost half a century ago. SCS is inspired by the gate-control theory of pain proposed by Melzack and Wall (1). The traditional, paresthesia-based SCS utilizes tonic 40- to $60-\mathrm{Hz}$ stimulation that activates the dorsal column supplying the patient's painful region. However, conventional SCS has limitations such as distressing paraesthesia, limited clinical uses, inadequate pain inhibition, and progressive decline in effects over time (2).
Several years ago, a new SCS paradigm was developed for pain treatment, in which high-frequency SCS (HFSCS) is applied at low amplitudes so that the stimulation is subthreshold for sensory activation and does not lead to paraesthesia. The most common frequency used for $\mathrm{HF}-\mathrm{SCS}$ is $10 \mathrm{kHz}$ and is referred to as HF10-SCS. This is currently marketed as the $\mathrm{Nevro}^{\circledR} \mathrm{SCS}$ which was approved by the US Food and Drug Administration in 2015 (3).

Cochlear implants are surgically inserted prosthetic devices that utilize electrical stimulation to provide hearing. This is generally indicated for moderate to

From: 'Department of Anesthesiology, Pain Management, and Perioperative Medicine, Henry Ford Health System, Detroit, MI, USA; ${ }^{2}$ Department of Anesthesiology, University of Texas Medical Branch, Galveston, TX, USA

Corresponding Author: Ashwin Vaidyanathan, MD, E-mail: avaidya1@hfhs.org

Disclaimer: There was no external funding in the preparation of this manuscript.

Conflict of interest: Each author certifies that he or she, or a member of his or her immediate family, has no commercial association (i.e., consultancies, stock ownership, equity interest, patent/licensing arrangements, etc.) that might pose a conflict of interest in connection with the submitted manuscript.

Accepted: 2020-12-08, Published: 2021-06-14 
severe bilateral sensorineural hearing loss. The cochlear implant utilizes an external sound processor that transmits electrical signals from outside into an array of electrodes placed on the cochlea, which stimulates the cochlear nerve and produces hearing (4).

The input frequency range of a cochlear implant is typically between $100 \mathrm{~Hz}$ and $10 \mathrm{kHz}$. The HF10-SCS, which also operates at $10 \mathrm{kHz}$, theoretically poses a risk of interference with the cochlear implant's signal transmission. A literature search did not reveal any reported cases of SCS implantation in a patient with a cochlear implant. The following is our first-hand experience with HF10-SCS implantation in a patient with bilateral cochlear implants.

\section{CASE}

A 75-year-old White woman with a history of bilateral cochlear implants for severe sensorineural hearing loss presented to our chronic pain clinic with low back pain for over 10 years. The patient described the pain as electric shock-like, located in the lower back and radiating along the posterior aspect of the bilateral lower extremities to the toes, and associated with tingling and numbness. Physical exam revealed features of lumbosacral radiculopathy without any sensory or motor deficits. Magnetic resonance imaging of the spine could not be performed due to her cochlear implants. The patient underwent a computed tomography scan of the lumbosacral spine, which revealed L3-4, L4-5, and L5-S1 broad disc bulging with severe bilateral foraminal stenosis and compression of exiting L5 roots. The patient's treatment regimen consisted of nonsteroidal anti-inflammatory drugs, gabapentinoids including gabapentin, and muscle relaxants including methocarbamol, which provided mild relief. Not being a surgical candidate due to poor pulmonary reserve and age, the patient was scheduled for a lumbar epidural steroid injection. The patient had temporary, but significant (75\%-80\%) improvement in pain for an average of 4 weeks with lumbar epidural steroid injections on 3 occasions. After adequate psychological assessment, she was considered for a Nevro ${ }^{\circledR}$ SCS implant. There was concern regarding her bilateral cochlear implants and possible risk of interference with SCS. The patient's cochlear implants were placed in 2004 following drug-related severe bilateral sensorineural hearing loss. Her cochlear implant was manufactured by Cochlear Americas ${ }^{\circledR}$ using a cp 910 processor. A literature search did not reveal any reported cases of HF-SCS implantation in a patient with cochlear implants. We contacted the cochlear implant manufacturer (Cochlear Americas) and inquired regarding the feasibility and potential interference. Cochlear Americas provided a letter stating that as long as the spinal cord implant is more than $20 \mathrm{~cm}$ away from the cochlear implant, there should not be any interference. We scheduled her for a Nevro ${ }^{\circledR}$ SCS trial. The SCS trial was performed at the entry point of the L1-L2 interlaminar space and leads were positioned at the top of T8 (right lead) and the bottom of T8 (left lead) (Fig. 1).

In the postanesthesia care unit (PACU), the patient did not experience any interference with cochlear implant even at triple the average SCS stimulation strength. At the one-week follow-up, the patient reported excellent pain relief (approximately $80 \%$ ) and also stated that she had significant functional improvement including the ability to climb stairs and go grocery shopping. The SCS leads were removed and the patient then went on to undergo percutaneous SCS electrode placement with a buttock pulse generator. Following the electrode placement, the patient continues to describe excellent relief in her radiculopathy and reports optimum functioning of her cochlear implants. There was no interference in her hearing - no hiss, electrical noise, or variation in audio quality.

\section{DISCUSSION}

In this case report, we have successfully used HFSCS in a patient with cochlear implants for lumbar radiculopathy without any clinically significant auditory interference.

The HF10-SCS involves application of short-duration (30 $\mu \mathrm{s})$, high-frequency $(10 \mathrm{kHz})$, low-amplitude (1 to $5 \mathrm{~mA}$ ) pulses to the spinal epidural space via spinal epidural electrode arrays (leads) at vertebral levels corresponding with perceived pain (3).

The cochlear implant works by stimulating the neural tissue of the spiral ganglion through electrical impulses. The electrodes implanted within the cochlea provide stimulation by means of a series of bipolar current impulses whose amplitude and frequency are controlled by the speech processor. This stimulation thereby creates auditory sensations at the level of the cerebral cortex (4).

The cochlear implant operates at a frequency range of $100 \mathrm{~Hz}$ to $10 \mathrm{kHz}$, which poses a risk that the SCS impulses that operate at $10 \mathrm{kHz}$ may be wrongly interpreted as sound.

The FDA Premarket Approval for the Cochlear Ameri- 
cas Nucleus ${ }^{\circledast}$ cochlear implant system notes that there is a risk of interference with neuromodulation devices (e.g., SCS) - typically when kept within $1 \mathrm{~cm}$ (5). In our patient, with the SCS leads being implanted at a T8 level, there was a considerable distance between the leads and the cochlear implant.

Studies have shown successful usage of various devices that rely on electrical stimulation in patients with cochlear implants, including electrocautery (6-8), cardiac pacemaker (9), and deep brain stimulator (10). The most important factor found to relate to interference is the distance from the cochlear implant. Out of these devices, the only documented complication was described by Roberts et al (11), in which the electrosurgical unit (operating at level 7) that was being used for a dental procedure led to the destruction of the cochlear implant circuitry. The British Cochlear Implant Group advises that monopolar cautery should not be used in the head and neck region in patients with cochlear implants and that bipolar cautery should not be used within $2 \mathrm{~cm}$ of the implant (6).

Bianchin et al (12) published a report on successful cochlear implantation in a patient with a preexisting traditional (Medtronic) paresthesia-based SCS; the SCS had been previously implanted, but was inactive. Unlike the HF10-SCS, the traditional SCS generates low-frequency impulses in the range of 40 to $60 \mathrm{~Hz}$, which is technically below the operating frequency of the cochlear implant.

\section{REFERENCES}

1. 1. Melzack R, Wall PD. Pain mechanisms: A new theory. Science 1965; 150:971-979.

2. 2. Chakravarthy $\mathrm{K}$, Richter $\mathrm{H}$, Christo PJ, Williams K, Guan Y. Spinal cord stimulation for treating chronic pain: Reviewing preclinical and clinical data on paresthesia-free high-frequency therapy. Neuromodulation 2018; 21:10-18.

3. 2. Kapural L, Yu C, Doust MW, et al. Novel 10-kHz high-frequency therapy (HF10 Therapy) is superior to traditional low-frequency spinal cord stimulation for the treatment of chronic back and leg pain: The SENZA-RCT randomized controlled trial. Anesthesiology 2015; 123:851-860.

4. 3. Tognola G, Parazzini M, Sibella F, Paglialonga A, Ravazzani P. Electromagnetic interference and cochlear implants. Ann Ist Super Sanita 2007; 43:241-247.

5. 4. Center for Devices and Radiological Health, US Food and Drug Administration. Premarket approval application for the Nucleus 24 Cochlear Implant System by Cochlear Corporation. Rockville, MD: US Food and Drug Administration; 1998. FDA Premarket Approval (PMA) Number P970051.

6. 5. Frampton SJ, Ismail-Koch $\mathrm{H}$, Mitchell TE. How safe is diathermy in patients with cochlear implants? Ann R Coll

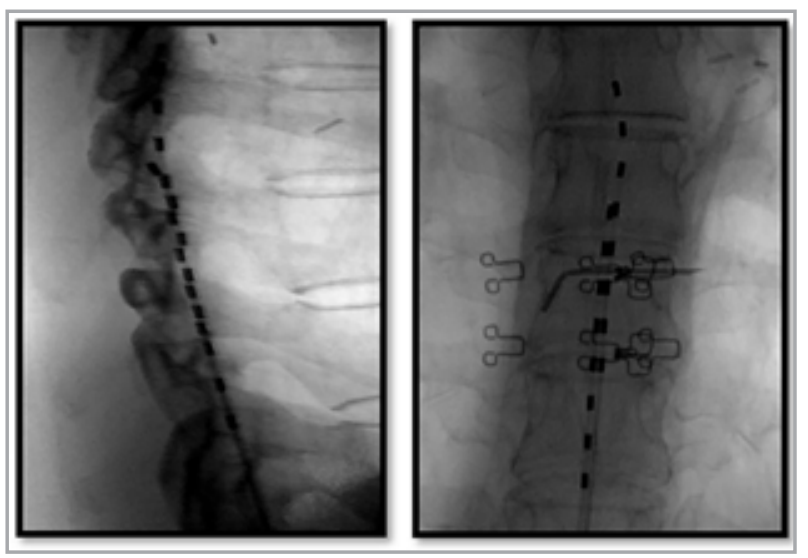

Fig. 1. SCS Trial - lead placement.

\section{CONCLUSION}

We successfully implanted an HF10-SCS at the thoracic level in a patient with bilateral cochlear implants without any auditory interference. Although these devices can theoretically lead to interference due to similarities in operating frequencies, in our experience, due to the significant distance between the cochlear implants and the thoracic leads, no clinical interference was detected. It remains to be seen if such interference may become evident with cervical leads.

This can potentially highlight the safety of using HFSCS in patients with other neuromodulation devices, notably the cochlear implant.

Surg Engl 2012; 94:585-587.

7. 6. Tien DA, Woodson EA, Anne S. Safety of monopolar electrocautery in patients with cochlear implants. Ann Otol Rhinol Laryngol 2016; 125:701-703.

8. 7. Jeyakumar A, Jackson NM, Givens VB, Brickman TM, Arriaga MA. Does electrocautery damage cochlear implants? Otol Neurotol 2015; 36:600-603.

9. 8. Triglia JM, Beliaeff M, Faugere G. Cochlear implantation in a pacemaker patient. Laryngoscope 1996; 106:11841186.

10. 9. De Los Reyes K, Chandrasekhar SS, Tagliati M, Alterman R. Successful implantation of a deep brain stimulator for essential tremor in a patient with a preexisting cochlear implant: Surgical technique: Technical case report. Neurosurgery 2010; 66:372; discussion 372.

11. 10. Roberts S, West LA, Liewehr FR, Rueggeberg FA, Sharpe DE, Potter BJ. Impact of dental devices on cochlear implants. J Endod 2002; 28:40-43.

12. 11 Bianchin G, Polizzi V. A case report on compatibility of electronic implants: Spinal cord stimulator and cochlear implant. Biochem Physiol 2014; 4:1. 
